\title{
Quorum sensing in the probiotic bacterium Escherichia coli Nissle 1917 (Mutaflor) - evidence that furanosyl borate diester (Al-2) is influencing the cytokine expression in the DSS colitis mouse model
}

Christoph A Jacobi ${ }^{1,4^{*}}$, Stefanie Grundler ${ }^{1}$, Chih-Jen Hsieh ${ }^{1}$, Julia Stefanie Frick ${ }^{2}$, Patrick Adam ${ }^{3}$, Georg Lamprecht ${ }^{1}$, Ingo B Autenrieth ${ }^{2}$, Michael Gregor ${ }^{1}$ and Peter Malfertheiner ${ }^{4}$

\begin{abstract}
Background: "Quorum sensing" (QS) is the phenomenon which allows single bacterial cells to measure the concentration of bacterial signal molecules. Two principle different QS systems are known, the Autoinducer 1 system (Al-1) for the intraspecies communication using different Acyl-homoserine lactones (AHL) and Al-2 for the interspecies communication. Aim of this study was to investigate QS of Escherichia coli Nissle 1917 (Mutaflor).

Results: While E. coli Nissle is producing Al-2 in a density dependent manner, no Al-1 was produced. To study the effect of Al-2 in the DSS (dextran sulphate sodium) induced mouse model of acute colitis, we silenced the corresponding gene luxS by intron insertion. The mutant bacterium E. coli Nissle::/ $/ u x S$ was equally effective in colonizing the colon and the mutation turned out to be 100\% stable during the course of the experiment. Isolating RNA from the colon mucosa and performing semiquantitative RT PCR, we were able to show that the expression of the pro-inflammatory cytokine IFN-y was suppressed in mice being infected with the E. coli Nissle wild type. Mice infected with the E. coli Nissle::/UxS mutant showed a suppressed expression of IL-10 compared to uninfected mice, while the expression of the pro-inflammatory cytokines IL-6 and TNF-a was higher in these mice. The expression of mBD-1 was suppressed in mice being infected with the mutant in comparison to the mice not infected or infected with the wild type. No differences were seen in the histological examination of the colon sections in the different groups of mice.
\end{abstract}

Conclusions: E. coli Nissle is producing Al-2 molecules, which are influencing the expression of cytokines in the mucosa of the colon in the DSS mice. However, if QS has a direct influence on the probiotic properties of E. coli Nissle remains to be elucidated.

Keywords: Quorum sensing, Escherichia coli Nissle, Autoinducer-2, DSS colitis, Cytokines

\footnotetext{
*Correspondence: christophjacobi@gmx.com

'Department of Internal Medicine I, University clinic Tübingen, Otfried

Müllerstr. 10, 72076, Tübingen, Germany

${ }^{4}$ Department for Gastroenterology, Hepatology and Infectiology, University

clinic Magdeburg, Leipzigerstr. 44, 39120, Magdeburg, Germany

Full list of author information is available at the end of the article
} 


\section{Background}

The communication of bacteria with each other is termed "quorum sensing" (QS) [1]. It is an important global gene regulatory mechanism, which is used by gram-negative as well as gram-positive bacteria, enabling individual bacteria to communicate and coordinate their behavior in populations. In general terms, it is often defined as cell density-dependent regulation of gene expression via extracellular signals. Bacteria produce small, diffusible signals, termed "autoinducers". When these signals reach a critical threshold concentration, the targeted QS genes are activated or repressed. Acyl-homoserine lactone signal-mediated QS systems are the primary QS system discovered in gram-negative bacteria. AHL were originally identified in marine bacteria, where they play a pivotal role in the regulation of bioluminescence in Vibrio fischeri [2-4]. Bacteria may produce one or more different AHL, which regulate diverse phenotypes, such as biofilm formation, swarming, production of proteases, antibiotics, siderophores or bioluminescence, conjugation, the modulation of the immune system and the induction of apoptosis [5-11]. While AHL are responsible for intraspecies communication, a second Autoinducer (AI-2) was discovered, which is responsible for the interspecies communication; furanosyl borate diester is synthezised by the gene $\operatorname{luxS}$, which is found in the genome of many gramnegative as well as gram-positive bacteria. It is an integral component of the activated methyl cycle, which detoxifies S-adenosyl-L-Methionine (SAM) [12-14].

Probiotics are, according to the FAO/WHO "live bacteria which when administered in adequate amounts confer to a health benefit to the host". The numerous mechanisms of the probiotic acting microorganisms include induced expression of certain cytokines, as well as increased secretion of immunoglobulin A and mucin, activation of lymphocytes and macrophages and inhibition of the adhesion and invasion of epithelial cells [15]. Escherichia coli Nissle 1917 (Mutaflor) is one of the most extensively studied probiotic bacterium. It was isolated in 1917 by Prof. Nissle from feces from a soldier during the First World War, who did not suffer from diarrhoea as his comrades did. Prof. Nissle realized the potential health benefits early on [16]. In addition, several important discoveries were made during recent years: $E$. coli Nissle is inducing human $B$-defensin 2 (hBD-2) expression in the cell culture in a time and density dependent manner [17]. In another study it has been shown that E. coli Nissle is decreasing TNF- $\alpha$ secretion [18]. Also, E. coli Nissle is outcompeting several nonpathogenic and pathogenic bacteria including ETEC and EPEC strains in biofilm formation [19]. Maintaining remission in ulcerative colitis using $E$. coli Nissle have shown equivalent efficacy to the gold standard mesalazine [20-22]. However, the exact mechanisms by which $E$. coli Nissle is exerting its beneficial effects are still not completely understood [23].

If $E$. coli Nissle is using QS to communicate with itself or with other species has not been elucidate so far. In this report we want to elucidate whether or not this bacterium is using QS. For the first time we were able to show that $E$. coli Nissle is producing AI-2 in a density dependent manner. We silenced the luxS gene by intron insertion and studied the effect of QS in the DSS mouse model of acute colitis. We observed differential expression of different cytokines and mBD-1, suggesting that indeed QS is influencing the probiotic properties of E. coli Nissle.

\section{Materials and methods \\ Animals}

Female C57BL/6 J (B6) mice (6-8 weeks of age) were purchased from Harlan Winkelmann (Borchen, Germany) and were kept under SPF conditions. The animals were handled in strict accordance with good animal practice and all animal work was approved by an appropriate institutional review committee (Anzeige vom 01.05.2006 Regierungspräsidium Tübingen).

\section{Bacterial strains and growth conditions}

The Escherichia coli strain Nissle 1917 (Mutaflor) was kindly provided to us by Dr. Sonnenborn from Ardeypharm (Herdecke, Germany). The luxS mutant of E. coli Nissle 1917 (E. coli Nissle::luxS) was generated by intron II insertion using the Targetron System (Sigma Aldrich, Munich, Germany). Using the sequence of luxS, PCR primers (Table 1.) were generated to mutate the intron. The mutated intron was cloned into the supplied vector pACD4K, which was transferred into $E$. coli Nissle. The "target site"(insertion site for the intron) of the luxS gene was determined to be the following sequence: CGAT ATCTCGCCAATGGGCTGCCGCACCGG - intron TTTTTATATGAGTCT. The correct insertion of the intron in the luxS gene was confirmed by PCR (see Additional file 1). In addition, experiments were conducted showing that AI-2 was not produced any longer by its mutant. The strains were routinely grown in Luria-Bertani (LB) broth at $37^{\circ} \mathrm{C}$, kanamycin $(50 \mu \mathrm{g} / \mathrm{ml})$ was added when growing the mutant.

For the mouse experiments, the bacteria were grown to an $\mathrm{OD}_{600}=1\left(\sim 0.8-1.2 \times 10^{9} \mathrm{CFU} / \mathrm{ml}\right)$ from an overnight culture diluted 1:100 in LB broth. The bacteria were collected by centrifugation, washed once in PBS and were resuspended in $200 \mathrm{ml}$ drinking water (+ 4\% DSS in the course of the experiment) for the mice.

\section{Detection of QS molecules}

We attempted to isolate AI-1 molecules as it was described before [24]. Extraction of culture supernatant 
Table 1 Bacterial strains and primers used

\begin{tabular}{|c|c|}
\hline Name & Sequence $\left(5^{\prime} \rightarrow 3^{\prime}\right)$ \\
\hline \multicolumn{2}{|c|}{ Primer for E.coli Nissle Mutaflor } \\
\hline Mut7f & GACCAAGCGATAACCGGATG \\
\hline Mut8r & GTGAGATGATGGCCACGATT \\
\hline \multicolumn{2}{|c|}{ Primer for "Targetron"mutation: } \\
\hline \multirow[t]{2}{*}{ IBS } & AAAAAAGCTTATAATTATCCTTACTGCCCCACCGG \\
\hline & GTGCGCCCAGATAGGGTG \\
\hline \multirow[t]{2}{*}{ EBS1d } & CAGATTGTACAAATGTGGTGATAACAGATAAGTCC \\
\hline & ACCGGTTTAACTTACCTTTCTTTGT \\
\hline \multirow[t]{2}{*}{ EBS2 } & TGAACGCAAGTTTCTAATTTCGGTTGGCAGTCGAT \\
\hline & AGAGGAAAGTGTCT \\
\hline EBSuniv & CGAAATTAGAAACTTGCGTTCAGTAAAC \\
\hline \multicolumn{2}{|l|}{ RT PCR Primer: } \\
\hline GAPDHf & CCAGCCGAGCCACATCGCTC \\
\hline GAPDHr & ATGAGCCCCAGCCTTCTCCAT \\
\hline IFN-yf & TCAAGTGGCATAGATGTGGAAGAA \\
\hline IFN- $\gamma r$ & TGGCTCTTGCAGGATTTTCATG \\
\hline IL-6f & GAGGATACCACTCCCAACAGACC \\
\hline IL-6r & AAGTGCATCATCGTTGTTCATACA \\
\hline IL-10f & AGGCGCTGTCATCGATTTCTC \\
\hline IL-10r & TGGCCTTGTAGACACCTTGGTC \\
\hline$m B D-1 f$ & ATGAAAACTCATTACTTTCTCCTGG \\
\hline$m B D-1 r$ & ACTACTGTCAGCTCTTACAACA \\
\hline TNF-af & GCAAGCTTCGCTCTTCTGTCTACTGAACTTCGG \\
\hline TNF-ar & GCTCTAGAATGAGATAGCAAATCGGCTGACGG \\
\hline \multicolumn{2}{|l|}{ Bacteria: } \\
\hline DH5a [pSB403] & DH5a carrying plasmid pSB403 \\
\hline E. coli Nissle 1917 & DSM6601; Serovar 06:K5:H1 \\
\hline E. coli Nissle::/UxS & Nissle mutated in the luxS gene \\
\hline Vibrio harveyi BB120 & Vibrio harveyi wildtype \\
\hline Vibrio harveyi BB886 & Vibrio harveyi Al-1 Sensor \\
\hline Vibrio harveyi BB170 & Vibrio harveyi Al-2 Sensor \\
\hline
\end{tabular}

(50 ml, corresponding to $5 \times 10^{10}$ bacteria) yielded $500 \mu \mathrm{l}$ of AHL concentrate in ethyl acetate. Appropriate dilutions of the concentrates were loaded on a thin-layer chromatography (TLC) plate (RP- $18 \mathrm{~F}_{254 \mathrm{~S}} ; 20$ by $20 \mathrm{~cm}$; Merck, Darmstadt, Germany) and were run in a moisture chamber containing a mixture of $60 \%$ methanol and $40 \%$ distilled water for $6 \mathrm{~h}$ [25]. After the plate was dried, it was overlaid with $200 \mathrm{ml}$ of $0.6 \%$ soft LB agar seeded with $20 \mathrm{ml}$ of a logarithmically grown culture of the lux-based AHL biosensor strain E. coli [pSB403], which is able to respond to a range of different AHL by luciferase production (e.g., BHL, HHL, OHHL, and ODHL) [26]. The incubation was carried out overnight in a moisture chamber at $30^{\circ} \mathrm{C}$, and AHLs were detected via autoradiography.

Autoinducer-2 (AI-2) levels in cell-free culture supernatant were measured using the Vibrio harveyi bioluminescence assay as described previously [13]. Briefly, the bacteria were grown as described above and cell-free supernatant was prepared at different time points by centrifugation of the sample at $10.000 \times g$ for 10 minutes. The supernatant was immediately frozen at $-80^{\circ} \mathrm{C}$ until being subjected to AI measurements: a $60 \mu \mathrm{L}$ aliquot of each sample was added to $600 \mu \mathrm{l}$ of the sensor bacterium $V$. harveyi BB170 (AI-2) or $V$. harveyi BB886 (AI-1) (being diluted 1:5.000 after being grown in "AB" medium) [13] and was incubated at $30^{\circ} \mathrm{C}$. Every hour the bioluminescence was measured using a Wallac Luminometer (Freiburg, Germany). All measurements were reported at the three hour incubation period, when the difference between negative controls and positive controls reached maximal levels. The measurements with the DH5 $\alpha$ [pSB403] sensor bacterium was performed in a similar fashion, however the luminescence was measured after overnight incubation.

\section{Experiments with DSS colitis mice}

After bacterial decontamination of the intestine with streptomycin $(5 \mu \mathrm{g} / \mathrm{ml}$ in drinking water) for two days [27], the success of this procedure was monitored by plating out stool on blood agar, LB agar and McConkey Agar and incubating them for three days under aerobic and anaerobic conditions at $37^{\circ} \mathrm{C}$. From day three the bacteria were administered in the drinking water of the mice, which was replaced every two days. Starting at day five of the experiment, $4 \%$ of DSS was dissolved in the drinking water to induce acute colitis (Figure 1). The viability of the bacteria was not affected by the presence of the DSS, as was checked by plating the suspension on agar plates. The weight of each mouse was recorded every day and the whole experiment ended at day nine when the maximal loss of weight was $15 \%$ of their initial weight. The mice were killed in a $\mathrm{CO}_{2}$ containing atmosphere. The large intestine was removed under sterile conditions. The length of the colon was measured. Stool was weighted, resuspended, homogenized, diluted and plated on blood-, LB- and McConkey plates and were incubated in an anaerobic and aerobic atmosphere at $37^{\circ} \mathrm{C}$. To confirm that the isolated bacteria where indeed E. coli Nissle wild type or mutant, we performed colony PCR with Nissle specific primers (Table 1). For this we extracted bacterial DNA by suspending bacterial cell mass in steril water and incubating the suspension at $95^{\circ} \mathrm{C}$ for 10 minutes. After centrifugation $(5.000 \mathrm{rpm} ; 5$ minutes), the DNA containing supernatant was used for PCR [28]. The mouse experiments were repeated twice, each group of mice consisted of three to five mice. 


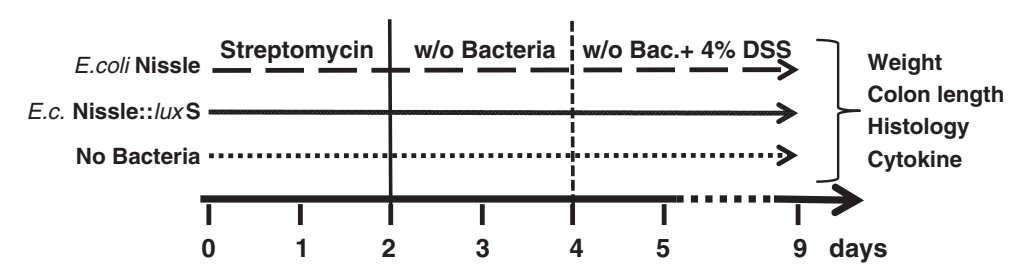

Figure 1 Experimental design of the DSS colitis model. The bacterial decontamination of the mouse intestine was performed by streptomycin treatment for the first two days of the experiment, followed by oral feeding with E. coli Nissle, E. coli Nissle::luxS or pure drinking water. Acute colitis was induced by administration of 4\% DSS in addition to the bacteria to the drinking water, starting from day five of the experiment.

\section{Expression of cytokines and mouse beta-defensin-1 from mucosa of the colon}

To analyze the cytokine and mouse beta defensin-1 (mBD-1) mRNA expression in the colonic mucosa, the mucosa (about $0.5 \mathrm{~cm}^{2}$ ) was carefully scraped off the colon. RNA was isolated using the RNAeasy Minikit (Qiagen, Hilden, Germany). Isolated RNA was reverse transcribed with Superscript reverse transcriptase (Invitrogen, Karlsruhe, Germany), oligodT- and random hexamer primers (Invitrogen). RT PCR was performed with the primers denoted in Table 1 using $25 \mathrm{ng}$ of cDNA template on an ABI Prism 7000 System using the SYBR green method (Fermentas, St. Leon Rot, Germany). Thermal cycling conditions were: $95^{\circ} \mathrm{C} / 10 \mathrm{~min}$ followed by 40 cycles of $92^{\circ} \mathrm{C} / 15 \mathrm{~s}$ and $60^{\circ} \mathrm{C} / 60 \mathrm{~s}$. Detection of fluorescent signal was performed according to the recommended protocols for the ABI Prism 7000 Real Time PCR machine (Applied Biosystems, Foster City, California, USA) The data was analyzed by the $\Delta \mathrm{C}_{\mathrm{T}}$ method using GAPDH for normalization $\left(\Delta \mathrm{C}_{\mathrm{T}}=\mathrm{C}_{\mathrm{T}}^{\text {sample }}\right.$ $\left.-\mathrm{C}_{\mathrm{T}}^{\mathrm{GAPDH}}\right)$. For each increase in $\Delta \mathrm{C}_{\mathrm{T}}(\mathrm{x})$, the expression is increased by a factor of $2^{\mathrm{x}}[29]$.

\section{Histological scoring}

Colon tissue was fixed in ice-cold neutral buffered $4 \%$ formalin for at least two hours followed by a washing step in phosphate buffered saline. For cryoprotection the tissue was treated in a $30 \%$ saccharose solution overnight. Afterwards, the tissue was embedded in paraffin and cut into $2 \mu \mathrm{m}$ sections. Samples were stained with hematoxylin and eosin (H\&E) (Merck, Darmstadt, Germany). Sections were analyzed in a blinded fashion by one pathologist.

\section{Statistical analysis}

All data are presented as means \pm S.D. Analysis of variance and students $t$ tests were applied when appropriate.

\section{Results}

Al-1/AI-2 production in E. coli Nissle 1917

To elucidate whether or not $E$. coli Nissle is communicating via QS molecules, we attempted to detect QS signal molecules. In case of AI-1, we tried to isolate and detect homoserine lactones. The direct isolation of AHL from the culture supernatant failed (data not shown), as did the indirect detection via two different sensor strains, DH5 $\alpha$ [pSB403] and Vibrio harveyi BB886 (Figure 2A; B): while the number of bacteria were increasing during the growth phase as shown by the lines in the diagrams, the luminescence emitted by the sensor strains was low. Eventhough the luminescence was doubled after six hours of incubation, it still remained at background level. However, when we attempted to detect AI-2 from the supernatant of the bacterial cultures, we were successful. Employing the AI-2 sensor strain Vibrio harveyi BB170, we detected furanosyl borate diester in a density dependent manner: after four hours of incubation, the luminescence was more than six times the amount (in comparison to the two hours incubation period). And after six hours the luminescence was 10-fold higher. Thus, for the first time, we are able to show that $E$. coli Nissle is producing AI-2. In the logarithmic and late logarithmic phase of the growth curve, the production of AI- 2 reached its maximum. Here the luminescence was more than 10-fold higher than the luminescence of the AI-1 sensor strain Vibrio harveyi BB886 (Figure 2C). During the later phases of growth, the AI-2 production is leveling off again (data not shown). Using specific primers derived from the E. coli K12 luxS sequence (Table 1), we were able to amplify the $l u x S$ gene from $E$. coli Nissle (data not shown).

In order to study the effect of AI-2 of E. coli Nissle in the DSS mouse model of acute colitis, we mutated the luxS gene by intron insertion. Genotypic and phenotypic control experiments confirmed the mutation: the intron II was inserted into the luxS gene. No AI-2 was detected in the supernatant of the mutant as compared to the corresponding wild type E. coli Nissle (see Additional file 1).

Colonisation and stability of $E$. coli Nissle and E. coli Nissle::IUxS in the DSS mouse model of acute colitis For the first time, we showed that E. coli Nissle is producing AI-2 molecules. In the next series of experiments, we 

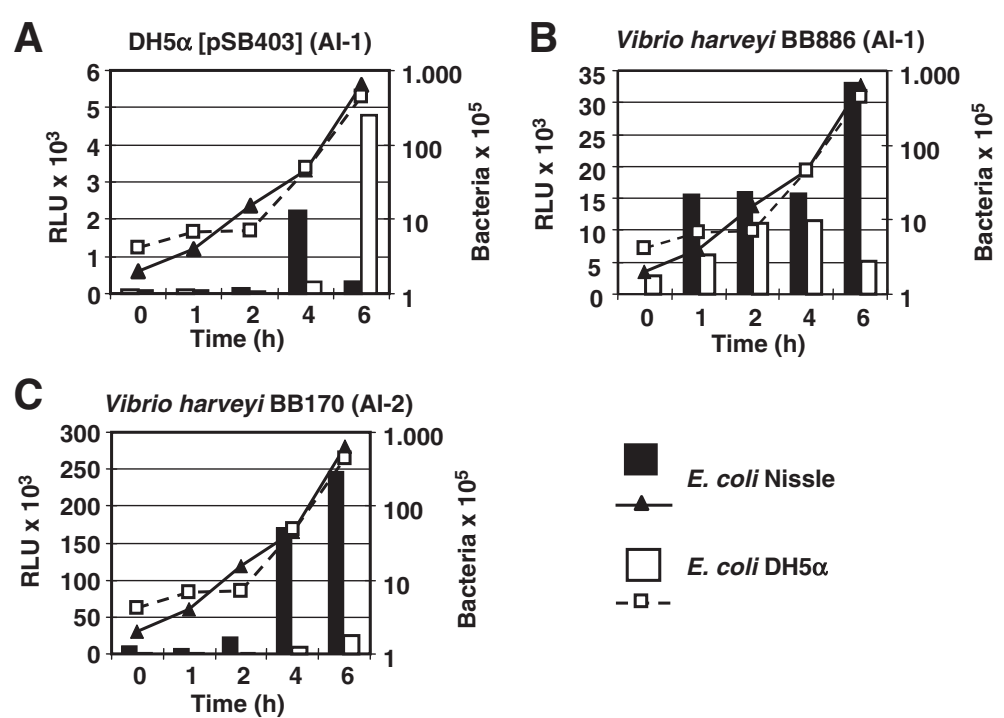

Figure $2 \mathrm{Al}-1$ and Al-2 production in E.coli Nissle and E.coli DH5a with the sensor bacteria E.coli DH5a [pSB403] (A), V. harveyi BB886 (B) and V. harveyi BB170 (C). Overnight cultures of E.coli Nissle and E.coli DH5a were diluted 1:100 in LB medium containing 0.5\% glucose. At the given time intervals aliquots were drawn, the bacterial numbers were determined by serial dilutions (lines; right Y-axis). Bacterial supernatants were obtained by centrifugation and frozen at $-80^{\circ} \mathrm{C}$. The presence of $\mathrm{Al}-1$ and $\mathrm{Al}-2$ was determined by incubating the respective sensor bacteria with the supernatants (via the luminescence (bars) - left Y-axis).

elucidated whether or not AI-2 produced by E. coli Nissle has an influence in mice. For this, we used a DSS model of acute colitis in female C57/BL6 mice. In a first step, we performed control experiments to elucidate the effect of the luxS mutation in comparison to the wild type on the colonization and stability in mice. The bacteria were supplied in the drinking water of the mice (Figure 3). The rate of colonization of $E$. coli Nissle and its correspondent E. coli Nissle::luxS mutant were very similar as was shown by diluting and plating the stool on different agar plates (Figure 3A). To confirm that the isolated bacteria are indeed $E$. coli Nissle, a PCR with Nissle specific primers was performed. The isolated clones from the plates were either $E$. coli Nissle wild type or the E. coli Nissle::luxS mutant; the Nissle specific PCR was positive, as shown by the a specific amplified PCR band (Figure 3B). In another series of experiments we confirmed the stability of the mutant in the mice: agar plates with and without the antibiotic kanamycin were inoculated with clones from mice, which were fed with the mutant. $100 \%$ of the clones were resistant to kanamycin confirming the stability of the luxS mutation in the mice during the course of the experiment of nine days.

\section{Effect of E. coli Nissle and E. coli Nissle::/uxS in the DSS mouse model of acute colitis}

The change in weight is a good and easy way to monitor the health status of mice. The development of colitis will lead to weight loss due to the inability to absorb nutrients in the intestine. Thus, the weight of each mouse was measured every day during the course of each experiment. Interestingly, the weight changes of the
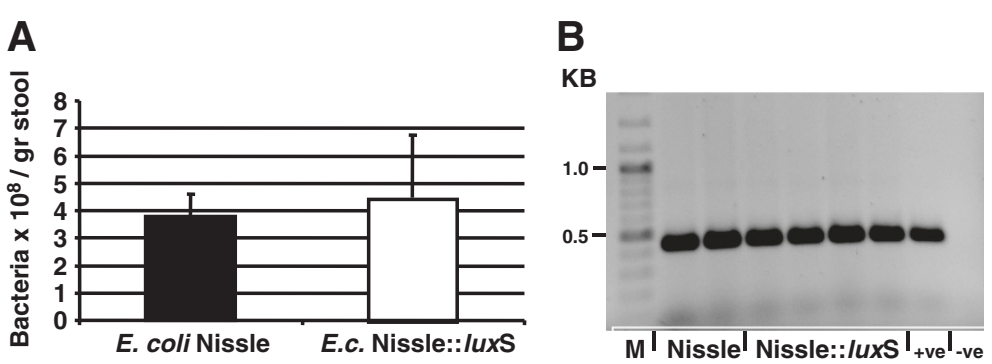

Figure 3 Control experiments for colonization (A) and stability (B). At the end of each experiment stool was weighted, homogenized, diluted and plated on agar followed by incubation at $37^{\circ} \mathrm{C}$ for three days. Bacterial colonies were counted (A). Colony PCR with Nissle specific primers (Table 1) was performed on selected bacterial clones. On the left is the molecular weight standard in kilobases (KB) (B). 
mice in the different groups were different. Surprisingly, the mice being fed with the wild type $E$. coli Nissle lost more weight on average than the mice being fed with the E. coli Nissle::luxS or the mice which received $4 \%$ DSS in their drinking water only. At the end of each experiment the average weight loss of the mice receiving the E. coli Nissle::luxS mutant or only 4\% DSS was similar. While their weight loss was $5 \%$, the average weight loss of the mice being fed with E. coli Nissle was 15\% (Figure 4). In respect to the fur and how the mice move, there was a significant difference between the groups of mice. The group infected with the wild type $E$. coli Nissle looked very sick, with their fur being scrubby and their movement around the cage being rather erratic.

The colon length is another valuable measurement to determine the severity of a colitis. During the course of colitis, the colon is shortened. Compared to the DSS control, the length of the colon from the mice being infected with the E. coli Nissle::luxS was significant longer $(\mathrm{p}<0.05)$. The colon of the mice infected with the wild type E. coli Nissle was longer than the DSS control (moderate significance $\mathrm{p}<0.1$ ) (Figure 5).

\section{Expression of cytokines and mBD-1 in the DSS mice}

It has been shown by a number of authors, that $E$. coli Nissle is influencing the expression of cytokines and defensins $[17,30]$. Here we elucidated, if AI-2 is influencing their expression. We have chosen a number of cytokines, which are well characterized, both pro- and antiinflammatory, and also the mouse defensine mBD-1 [31,32] for analysis. After oral infection of the mice with E. coli Nissle or its correspondent E. coli Nissle::luxS mutant, an acute colitis was induced by DSS. Expression of the pro-inflammatory cytokine Interferon- $\gamma$ was suppressed $32-64$ fold $\left(2^{5}-2^{6}\right)$ in mice infected with the

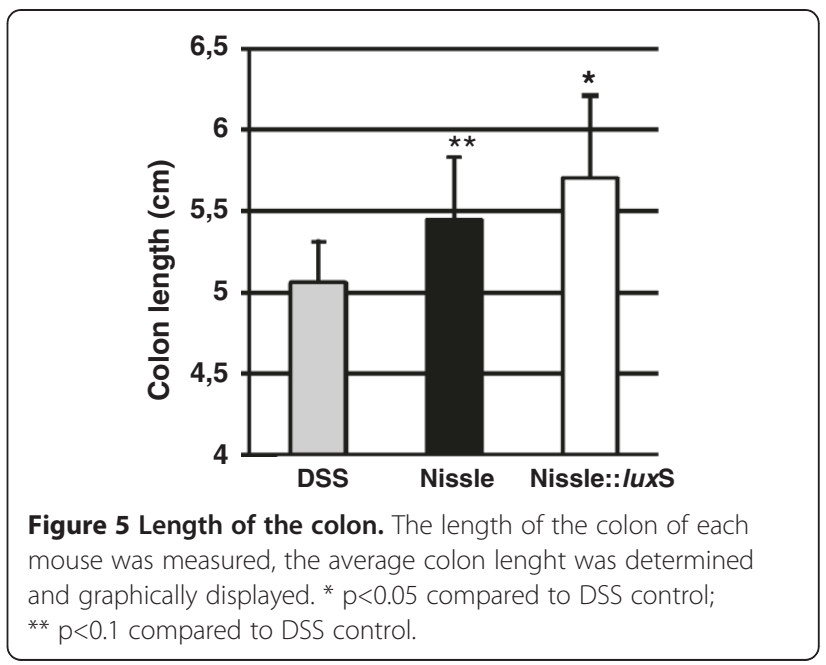

E. coli Nissle wild type in comparison to the mice being infected with $E$. coli Nissle::luxS or not being infected at all. Significant suppression of the anti-inflammatory cytokine IL-10 in mice being infected by the mutant in comparison to the uninfected mice was seen. The suppression was, however not significant in comparison to the mice been fed with the wild type. In the respect to the pro inflammatory cytokines IL- 6 and TNF- $\alpha$ an increased expression of about 8 fold was seen in mice infected with the mutant in comparison with the DSS control (moderate significance of $\mathrm{p}<0,1$ ) and the wild type. The expression of the mBD-1 was suppressed about 4 fold in mice being infected with the luxS mutant in comparison to the wild type infected mice (moderate significance of $\mathrm{p}<0.1$ ) (Figure 6). We also measured the expression of cytokines in mice which were not treated with DSS and bacteria. In these mice the expression of cytokines was suppressed (data not shown).

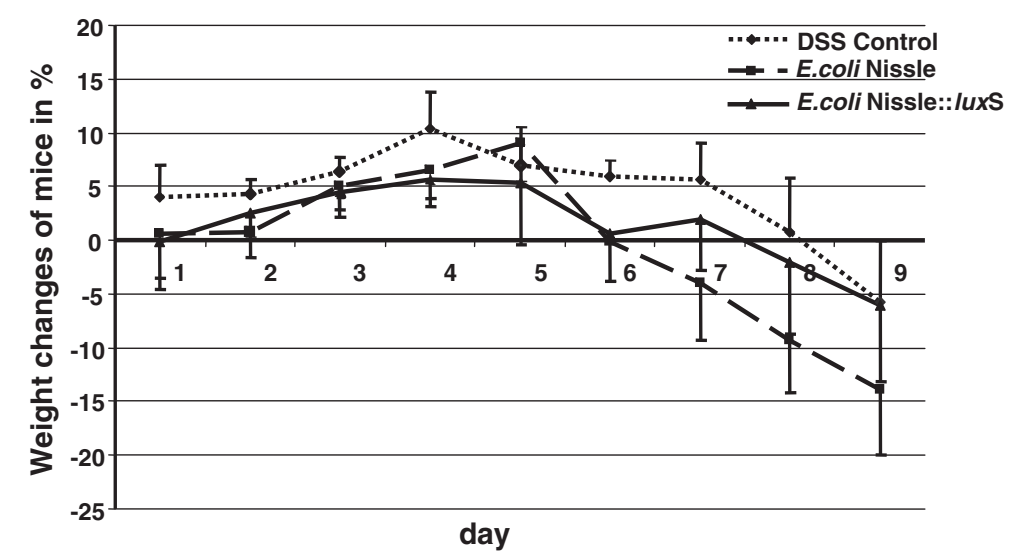

Figure 4 Changes in body weight in the course of the experiment. The body weight of each mouse was determined every day during the course of each experiment. " $0 \%$ " was the weight of the mice, when we started the experiment, the gain/loss of the weight was determined as a percentage value. 


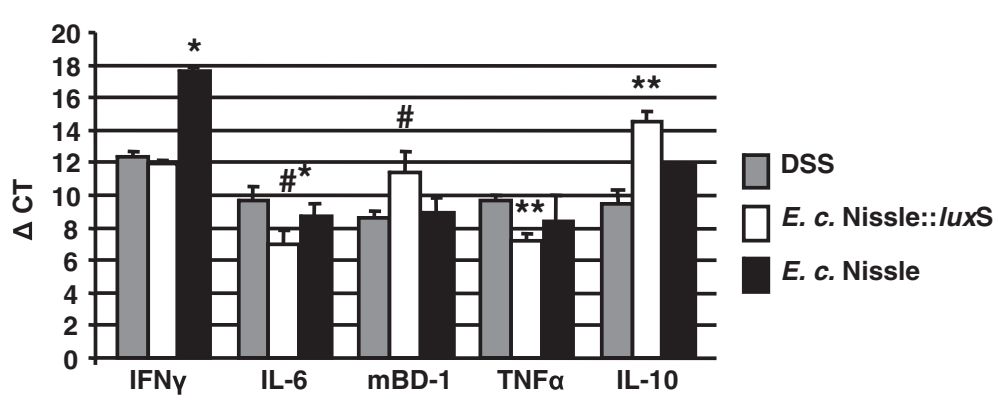

Figure 6 Expression of cytokines and mBD-1 in the colon. After scraping the mucosa from the colon, RNA was isolated, transcribed and a semiquantitative RT PCR was run using the primers despicted in Table 1. The $\Delta C T$ values were determined and graphically displayed. ${ }^{*} p<0.05$ compared to E. coli Nissle::/uxS and DSS control; ** $p<0.05$ compared to DSS control; \# $p<0.1$ compared to Nissle; \#* $p<0.1$ compared to DSS control.

Histologic examination of the colon from the DSS mice The histology of the colon was examined by a single pathologist in a blinded fashion. A scoring system was used to evaluate the inflammation. Regions of patchy inflammations were seen, as denoted by infiltration of granulocytes (see star). Taken together, no significant differences in the pathology between the different groups of mice were seen (Figure 7).

\section{Discussion}

E. coli Nissle 1917 is a well established probiotic bacterium. Since its first isolation and description 95 years ago, the interest in this bacterium has increased steadily. Especially during the last few years, the understanding on the mechanisms this bacterium is employing for its beneficial effects, has increased substantially [23]. However, the exact mechanism of action still remains to be elucidated to completely "understand" this bacterium. One important gene regulation mechanism, QS, has, so far, not been looked at in E. coli Nissle. It is a density dependent genetic regulatory mechanism which allows single bacterial cells to measure the concentration of certain bacterial signal molecules. In case that $E$. coli Nissle is using QS, we would like to study its influence on the probiotic properties of the bacterium.

Not surprisingly, this bacterium, as all other $E$. coli strains having been studied so far, is not synthesizing homoserine lactones (AI-1 molecules) [13]. However, we showed for the first time, that AI-2 is produced by E. coli Nissle in a density dependent manner. During the logarithmic and late logarithmic growth phase the amount of AI-2 being produced is the largest. This finding suggests, that $E$. coli Nissle is using interspecies communication and is "talking" with other bacterial
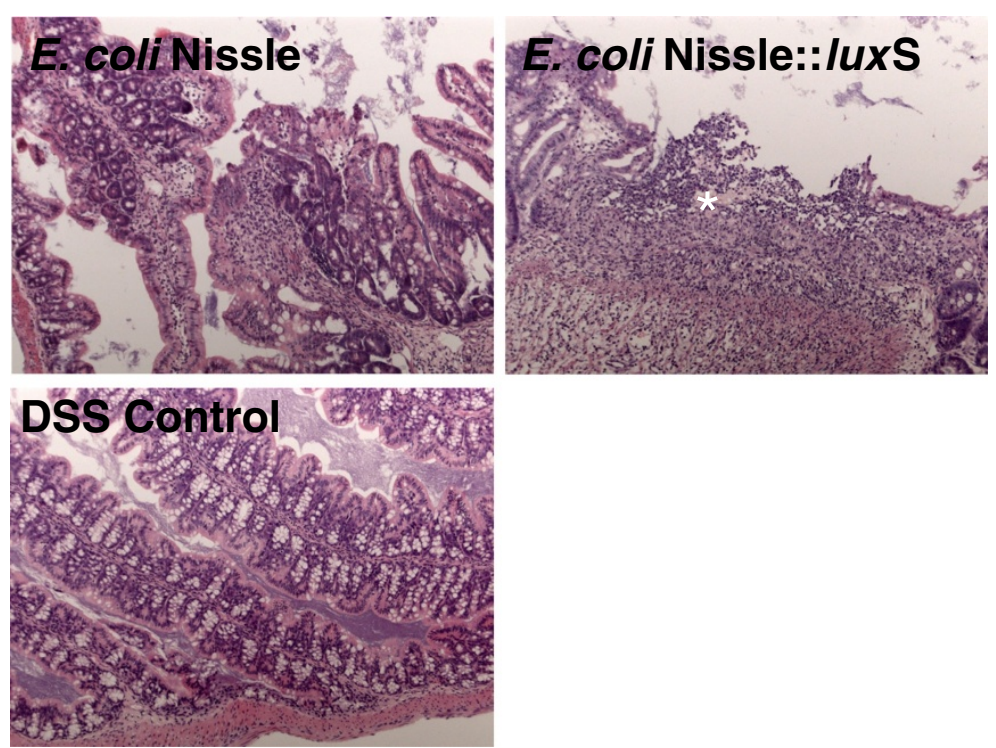

Figure 7 Histology of the colon. The paraffin embedded sections of the colon were stained with $\mathrm{H} \& \mathrm{E}$ and were viewed in a blinded fashion by a single pathologist. Patchy inflammation is marked by a star. 
species. In order to study the effect of AI-2 in E. coli Nissle, we silenced the corresponding gene via intron insertion. We performed control experiments and were able to show, that no AI- 2 was produced by the mutant any longer.

We studied the effect of luxS silencing of $E$. coli Nissle in the DSS mouse model of acute colitis. The wild type E. coli Nissle and the E. coli Nissle::luxS mutant behaved similar in respect to colonisation and stability, which is essential in animal experiments. Thus, AI-2 in E. coli Nissle is not necessary for survival of the bacterium, otherwise we would not have been able to isolate the mutant bacterium after days in the mouse intestine. Measuring the body weight of the mice during the course of the experiments showed that the mice which were fed with the wild type $E$. coli Nissle lost $15 \%$ of their initial weight. The other two groups of mice (E. coli Nissle:::luxS; DSS control) lost on average only $5 \%$ of their weight. In addition, the group of mice fed with the E. coli Nissle started to lose weight about two days early than the other two groups. In accordance with these data, these mice looked sicker: their fur was scrubby and their movement around the cages was rather erratic. We can speculate, that $E$. coli Nissle is fitter than its mutant, which makes the bacterium more prone for potential translocation: while under healthy conditions there is minimal translocation of intestinal bacteria in mesenterial lymph nodes, under inflammatory conditions the epithelial barrier is broken down and intestinal bacteria and also E. coli Nissle are translocated through the Peyer patches and the MLN [33]. The length of the colon is proportional to the level of its inflammation: increasing levels of inflammation results in shortening of the colon. The colon of these mice, which received only DSS, were the shortest, while the colon of the mice, which were inoculated with E. coli Nissle or its corresponding mutant were longer. To elucidate the effect of the oral infection of mice with $E$. coli Nissle wild type or its corresponding luxS mutant on the expression of cytokines, we choose four well characterized pro- and antiinflammatory cytokines and one defensine. In mice, which were fed with the wild type E. coli Nissle, the proinflammatory cytokine IFN- $\gamma$, was suppressed in the colon mucosa, while the anti-inflammatory cytokine IL10 was suppressed by $E$. coli Nissle::luxS. The expression of the pro-inflammatory cytokines Il- 6 and TNF- $\alpha$ was around 8 fold higher in mice infected with the $l u x \mathrm{~S}$ mutant, than in mice of the other two groups. On the other hand the expression of mBD-1 was suppressed in the mice infected with the mutant, in comparison to the mice which were infected with $E$. coli Nissle or were not infected at all. Taken together, we observed significant differences in the expression of cytokines between the different groups of mice. Eventhough the results were partly only moderately significant $(\mathrm{p}<0.1)$, we have generated evidence that AI-2 from E. coli Nissle is indeed influencing the expression of cytokines and defensins and thus may influence the probiotic properties. It is well known that E. coli Nissle is capable of inducing anti-inflammatory cytokines. Early on it was shown, that $E$. coli Nissle is stimulating the epithelial defense in Caco-2 cells [17]. The same authors showed that patients with Crohns disease, who have a mutation in NOD2 have a low level of expression of the defensins HD-5 and HD-6 [17]. The normal colon mucosa is producing human beta-defensin-1 (hBD-1; the homolog to mBD-1) [34]. The functional importance of defensins was shown in elegant experiments with HD-5 expressing transgenic mice. These mice became resistant towards an infection with salmonellae [35]. While pathogenic bacteria seem to suppress the production of defensins probably for self-defense [36], E. coli Nissle is protecting its host by defensin induction $[17,34]$.

It has been shown in a number of studies that homoserine lactones (AI-1) regulate the expression of cytokines and virulence factors, for example in Vibrio cholerae, Pseudomonas aeruginosa or EHEC [37-39]. However, the influence of AI-2 on the cytokine expression was discussed only in two reports so far $[40,41]$. Using microarrays the group showed the differential regulation of a number of genes involved in the complement pathway, regulation of cytokine expression and antigen presentation when infecting RAW264.7 macrophages with the wild type Vibrio vulnificus or with the corresponding lux $\mathrm{S}$ mutant.

Is QS also used by other probiotic bacteria? Studies were published only with bacteria of the genus Lactobacillus. Early on it was shown that Lactobacillus rhamnosum GG is communicating via AI-2 molecules [42]. It was shown that the $\operatorname{luxS}$ gene has a clear role in the acidic stress response; AI-2 activity increased by lowering the $\mathrm{pH}$ in a dose dependent manner [43]. A second probiotic bacterium, $L$. acidophilum strain La-5, showed that its supernatant is influencing the AI-2 concentration and the expression of virulence genes of the enterohemorrhagic E. coli (EHEC) 0157:H17 in the gut, inhibiting its colonization. In addition it was shown, that the supernatant is reducing the attaching and effacing lesions in HeLa cells. Also a significant inhibition of bacterial adhesion in Hep-2 cells was observed. In elegantly designed mouse experiments, the authors used slowscan CCD cameras to show reduced fecal shedding of luminescent EHEC constructs when L. acidophilus was fed additionally [44,45]. Another Lactobacillus species, L. plantarum was used successfully to inhibit the pathogenic activity of Pseudomonas aeruginosa. The AHL production, as well as the production of elastase and biofilm was inhibited by $L$ plantarum cultures and filtrates, but 
not by isolated, washed cells [46]. In another set of experiments with $L$. plantarum, it was shown that its LamBDCA quorum sensing system is responsible for the modulation of cytokine response in human PBMC [47]. These studies showed that indeed QS is influencing the probiotic properties of the studied Lactobacillus species. In the future, QS in other probiotic species should be studied, to hopefully confirm that QS is indeed influencing the probiotic properties.

Examining the histologic sections of the colon of the different groups of mice, we did not observe any significant difference between the groups. In contrast to the histopathology of a chronic inflammation, E. coli Nissle has no influence in case of an acute DSS induced colitis. Here one can observe histologic patchy mucosal damage with the loss of crypts, followed by the acute transmural infiltration of inflammatory cells. No T- or B-cells are necessary [48].

In this report we showed for the first time that the probiotic bacterium E. coli Nissle is producing AI-2 molecules. Here we show that AI-2 is affecting the regulation of cytokine expression in the DSS mouse model of acute colitis. In comparison to the E. coli Nissle luxS mutant, the mice which were infected with the wild type lost more weight, looked sicker regarding their fur and their lack of movement. On the other hand, mice, which were infected with the E. coli Nissle::luxS mutant showed a higher expression of pro inflammatory cytokines, but a reduced expression of the anti-inflammatory cytokine IL-10 or the mBD-1. Thus, it remains to be seen if AI-2 is influencing the probiotic properties of this important bacterium.

\section{Additional file}

Additional file 1: Construction of the E.coli Nissle::/uxS mutant; including control experiments: A: Schematic diagram of the construction of the mutant: left: E.coli Nissle wild type, right: E.coli Nissle::IuxS mutant. B: control PCR: Intron is inserted into the luxS gen (the different colored arrows are symbolizing different primer pairs) $\mathrm{M}$ : Marker; numbers are in kilobases. C: E.coli Nissle wild type (WT) produces $\mathrm{Al}-2$ while the E.coli Nissle::/uxS mutant does not.

\section{Competing interest}

The authors declare that they have no competing interests.

\section{Authors'contribution}

CAJ designed and performed most of the experiments. He supervised the experiments which were not performed by him. He has written the manuscript. The work of SG focused on the in vitro experiments while C-JH performed parts of the RT PCR and gave valuable advice. JSF introduced CAJ into the DSS mouse model and was of great help concerning the mouse experiments. PA was examining the histological sections. GL, IBA, MG and PM gave valuable advice concerning the experiments and for the preparation of the manuscript. All authors read and approved the manuscript.

\section{Author details}

'Department of Internal Medicine I, University clinic Tübingen, Otfried Müllerstr. 10, 72076, Tübingen, Germany. ${ }^{2}$ Institute of Medical Microbiology and Hygiene, University clinic Tübingen, Otfried Müllerstr. 10, 72076,

Tübingen, Germany. ${ }^{3}$ Institute for Pathology and Neuropathology, University clinic Tübingen, Otfried Müllerstr. 10, 72076, Tübingen, Germany.

${ }^{4}$ Department for Gastroenterology, Hepatology and Infectiology, University clinic Magdeburg, Leipzigerstr. 44, 39120, Magdeburg, Germany.

Received: 6 July 2012 Accepted: 9 July 2012

Published: 3 August 2012

\section{References}

1. Fuqua WC, Winans SC, Greenberg E: Quorum sensing in bacteria: the luxRLuxl family of cell density-responsive transcriptional regulators. J Bacteriol 1994, 176:269-275.

2. Nealson KH, Platt K, Hastings JW: Cellular control of the synthesis and activity of the bacterial luminescent system. J Bacterio/ 1970, 104:313-322.

3. Eberl $\mathrm{L}: \mathrm{N}$-acyl homoserine lactone-mediated gene regulation in gramnegative bacteria. Syst Appl Microbiol 1999, 22:493-506.

4. Miller MB, Bassler BL: Quorum sensing in bacteria. Annu Rev Microbiol 2001, 55:165-199.

5. Atkinson S, Throup JP, Stewart GS, Williams PA: Hierarchical quorumsensing system in Yersinia pseudotuberculosis is involved in the regulation of motility and clumping. Mol Microbiol 1999, 33:1267-1277.

6. Atkinson S, Chang CY, Sockett RE, Cámara M, Williams P: Quorum sensing in Yersinia enterocolitica controls swimming and swarming motility. J Bacteriol 2006, 188:1451-1461.

7. Dong $Y H, X u J L, L i X Z$, Zhang $L H:$ AiiA, an enzyme that inactivates the acylhomoserine lactone quorum-sensing signal and attenuates the virulence of Erwinia carotovora. Proc Natl Acad Sci U S A 2000, 97:3526-3531.

8. Hussain MB, Zhang $H B$, Xu JL, Liu Q, Jiang Z, Zhang LH: The acyl-homoserine lactone-type quorum-sensing system modulates cell motility and virulence of Erwinia chrysanthemi pv. zeae. J Bacteriol 2008, 190:1045-1053.

9. Li L, Hooi D, Chhabra SR, Pritchard D, Shaw PE: Bacterial N-acylhomoserine lactone-induced apoptosis in breast carcinoma cells correlated with down-modulation of STAT3. Oncogene 2004, 23:4894-4902.

10. Williams P, Camara M, Hardman A, Swift S, Milton D, Hope VJ: Quorum sensing and the population-dependent control of virulence. Phil Trans $R$ Soc Lond B Biol Sci 2000, 355:667-680.

11. Zhu J, Miller MB, Vance RE, Dziejman M, Bassler BL, Mekalanos JJ: Quorumsensing regulators control virulence gene expression in Vibrio cholerae. Proc Natl Acad Sci U S A 2002, 99:3129-3134.

12. Schauder S, Shokat K, Surette MG, Bassler BL: The LuxS family of bacterial autoinducers: biosynthesis of a novel quorum-sensing signal molecule. Mol Microbiol 2001, 41:463-476.

13. Surette MG, Bassler BL: Quorum sensing in Escherichia coli and Salmonella typhimurium. Proc Natl Acad Sci U S A 1998, 95:7046-7050.

14. Surette MG, Miller MB, Bassler BL: Quorum sensing in Escherichia coli, Salmonella typhimurium, and Vibrio harveyi: a new family of genes responsible for autoinducer production. Proc Natl Acad Sci U S A 1999, 96:1639-1644

15. McNabb B, Isakow W: Probiotics for the prevention of nosocomial pneumonia: current evidence and opinions. Curr Opin Pulm Med. 2008, 14:168-175.

16. Nissle A: Old and new experiences on therapeutic successes by restoration of the colonic flora with mutaflor in gastrointestinal diseases. Med Welt 1961, 29-30:1519-1523.

17. Wehkamp J, Harder J, Wehkamp K, Wehkamp-von Meissner B, Schlee M, Enders C, Sonnenborn U, Nuding S, Bengmark S, Fellermann K, Schroder JM, Stange EF: NF-KB- and AP-1-mediated induction of human beta defensin2 in intestinal epithelial cells by Escherichia coli Nissle 1917: a novel effect of a probiotic bacterium. Infect Immun 2004, 72:5750-5758.

18. Grabig A, Paclik D, Guzy C, Dankof A, Baumgart DC, Erckenbrecht J, Raupach B, Sonnenborn U, Eckert J, Schumann RR, Wiedenmann B, Dignass AU, Sturm A: Escherichia coli strain Nissle 1917 ameliorates experimental colitis via tolllike receptor 2- and toll-like receptor 4-dependent pathways. Infect Immun 2006, 74:4075-4082. 
19. Hancock V, Dahl D, Klemm P: Probiotic Escherichia coli strain Nissle 1917 outcompetes intestinal pathogens during biofilm formation. J Med Microbiol, 59:392-399.

20. Kruis W, Schutz E, Fric P, Fixa B, Judmaier G, Stolte M: Double-blind comparison of an oral Escherichia coli preparation and mesalazine in maintaining remission of ulcerative colitis. Aliment Pharmacol Ther 1997 , 11:853-858

21. Kruis W, Fric P, Pokrotnieks J, Lukas M, Fixa B, Kascak M, Kamm MA, Weismueller J, Beglinger C, Stolte M, Wolff C, Schulze J: Maintaining remission of ulcerative colitis with the probiotic Escherichia coli Nissle 1917 is as effective as with standard mesalazine. Gut 2004, 53:1617-1623.

22. Rembacken BJ, Snelling AM, Hawkey PM, Chalmers DM, Axon AT: Nonpathogenic Escherichia coli versus mesalazine for the treatment of ulcerative colitis: a randomised trial. Lancet 1999, 354:635-639.

23. Jacobi CA, Malfertheiner P: Escherichia coli Nissle 1917 (Mutaflor): new insights into an old probiotic bacterium. Dig Dis 2011, 29:600-607.

24. Geisenberger O, Givskov M, Riedel K, Hoiby N, Tummler B, Eberl L: Production of $\mathrm{N}$-acyl-L-homoserine lactones by $P$. aeruginosa isolates from chronic lung infections associated with cystic fibrosis. FEMS Microbiol Lett 2000, 184:273-278.

25. Shaw PD, Ping G, Daly SL, Cha C, Cronan JE Jr, Rinehart KL, Farrand SK: Detecting and characterizing $\mathrm{N}$-acyl-homoserine lactone signal molecules by thin-layer chromatography. Proc Natl Acad Sci U S A 1997, 94:6036-6041.

26. Winson MK, Camara M, Latifi A, Foglino M, Chhabra SR, Daykin M, et al: Multiple $\mathrm{N}$-acyl-L-homoserine lactone signal molecules regulate production of virulence determinants and secondary metabolites in Pseudomonas aeruginosa. Proc Natl Acad Sci U S A 1995, 92:9427-9431.

27. Conway T, Krogfelt KA, Cohen PS: The life of commensal Escherichia coli in the mammalian intestine. In EcoSal-Escherichia coli and Salmonella: cellular and molecular biology. Edited by Curtiss R III. Washington, DC: ASM Press; 2004. Chapter 8.3.1.2., 29 December.

28. Saiki RK, Gelfand DH, Stoffel S, Scharf SJ, Higuchi R, Horn GT, Mullis KB, Erlich HA: Primer-directed enzymatic amplification of DNA with a thermostable DNA polymerase. Science 1988, 239:487-491.

29. Lamprecht G, Hsieh CJ, Lissner S, Nold L, Heil A, Gaco V, Schäfer J, Turner JR, Gregor M: Intestinal anion exchanger down-regulated in adenoma (DRA) is inhibited by intracellular calcium. J Biol Chem 2009, 284(29):19744-19753.

30. Hafez M, Hayes K, Goldrick M, Warhurst G, Grencis R, Roberts IS: The K5 capsule of Escherichia coli strain Nissle 1917 is important in mediating interactions with intestinal epithelial cells and chemokine induction. Infect Immun 2009, 77:2995-3003.

31. Cross ML, Ganner A, Teilab D, Fray LM: Patterns of cytokine induction by gram-positive and gram-negative probiotic bacteria. FEMS Immunol Med Microbiol 2004, 42:173-180.

32. Zaalouk TK, Bajaj-Elliott M, George JT, McDonald V: Differential regulation of beta-defensin gene expression during Cryptosporidium parvum infection. Infect Immun 2004, 72:2772-2779.

33. Borruel N, Carol M, Casellas F, Antolin M, de Lara F, Espín E, Naval J, Guarner F, Malagelada JR: Increased mucosal tumor necrosis factor a production in Crohn's disease can be downregulated ex vivo by probiotic bacteria. Gut 2003, 51:659-664.

34. Wehkamp J, Harder J, Weichenthal M, Müller O, Herrlinger KR, Fellermann K, Schröder JM, Stange EF: Inducible and constitutive beta-defensins are differentially expressed in Crohn's disease and ulcerative colitis. Inflamm Bowel Dis 2003, 9:215-223.

35. Salzman NH, Ghosh D, Huttner KM, Paterson Y, Bevins CL: Protection against enteric salmonellosis in transgenic mice expressing a human intestinal defensin. Nature 2003, 422:522-526.

36. Salzman NH, Chou MM, de Jong H, Liu L, Porter EM, Paterson Y: Enteric salmonella infection inhibits Paneth cell antimicrobial peptide expression. Infect Immun 2003, 71:1109-1115.

37. Sperandio V, Torres AG, Girón JA, Kaper JB: Quorum sensing is a global regulatory mechanism in enterohemorrhagic Escherichia coli 0157:H7 J Bacteriol 2001, 183:5187-5197.

38. Tateda K, Ishii Y, Horikawa M, Matsumoto T, Miyairi S, Pechere JC, et al: The Pseudomonas aeruginosa autoinducer N-3-oxododecanoyl homoserine lactone accelerates apoptosis in macrophages and neutrophils. Infect Immun 2003, 71:5785-5793.
39. Zheng J, Shin OS, Cameron DE, Mekalanos JJ: Quorum sensing and a global regulator TsrA control expression of type $\mathrm{VI}$ secretion and virulence in Vibrio cholerae. Proc Natl Acad Sci U S A 2010, 107:21128-21133.

40. Shin NR, Lee DY, Shin SJ, Kim KS, Yoo HS: Regulation of proinflammatory mediator production in RAW264.7 macrophage by Vibrio vulnificus luxS and smcR. FEMS Immunol Med Microbiol 2004, 41:169-176.

41. Shin NR, Lee DY, Yoo HS: Analysis of gene expression in mouse alveolar macrophages stimulated with quorum-sensing mutants of Vibrio vulnificus. Jpn J Infect Dis 2008, 61:402-406.

42. Lebeer S, De Keersmaecker SC, Verhoeven TL, Fadda AA, Marchal K, Vanderleyden J: Functional analysis of luxS in the probiotic strain Lactobacillus rhamnosus GG reveals a central metabolic role important for growth and biofilm formation. J Bacterio/ 2007, 189(3):860-871.

43. Moslehi-Jenabian S, Gori K, Jespersen L: Al-2 signalling is induced by acidic shock in probiotic strains of Lactobacillus spp. Int J Food Microbiol 2009, 135(3):295-302.

44. Medellin-Peña MJ, Wang H, Johnson R, Anand S, Griffiths MW: Probiotics affect virulence-related gene expression in Escherichia coli 0157:H7. Appl Environ Microbiol 2007, 73(13):4259-4267.

45. Medellin-Peña MJ, Griffiths MW: Effect of molecules secreted by Lactobacillus acidophilus strain La-5 on Escherichia coli 0157:H7 colonization. Appl Environ Microbiol 2009, 75(4):1165-1172.

46. Valdéz JC, Peral MC, Rachid M, Santana M, Perdigón G: Interference of Lactobacillus plantarum with Pseudomonas aeruginosa in vitro and in infected burns: the potential use of probiotics in wound treatment. Clin Microbiol Infect 2005, 11(6):472-479.

47. van Hemert S, Meijerink M, Molenaar D, Bron PA, de Vos P, Kleerebezem M, Wells JM, Marco ML: Identification of Lactobacillus plantarum genes modulating the cytokine response of human peripheral blood mononuclear cells. BMC Microbiol 2010, 10:293.

48. Cooper HS, Murthy SNS, Shah RS, Sedergran DJ: Clinicopathologic study of dextran sulfate sodium experimental murine colitis. Lab Investig 1993, 69:238-249.

doi:10.1186/1757-4749-4-8

Cite this article as: Jacobi et al:: Quorum sensing in the probiotic bacterium Escherichia coli Nissle 1917 (Mutaflor) - evidence that furanosyl borate diester (Al-2) is influencing the cytokine expression in the DSS colitis mouse model. Gut Pathogens 2012 4:8.

\section{Submit your next manuscript to BioMed Central and take full advantage of:}

- Convenient online submission

- Thorough peer review

- No space constraints or color figure charges

- Immediate publication on acceptance

- Inclusion in PubMed, CAS, Scopus and Google Scholar

- Research which is freely available for redistribution 